\title{
Las construcciones fraseológicas verbales argumentativas inglesas (VAC) en las maniobras de remolque maritimo: detección y representación de sus propiedades sintácticas y semánticas
}

\author{
MARÍA-ARACELI LOSEY-LEÓN \\ Departamento de Filología Francesa e Inglesa \\ Universidad de Cádiz \\ Escuela de Ingenierías Marina, Náutica y Radioelectrónica \\ Centro Andaluz Superior de Estudios Marinos \\ Polígono Río San Pedro s/n \\ 11510 Puerto Real (Cádiz) \\ E-mail: araceli.losey@uca.es
}

LAS CONSTRUCCIONES FRASEOLÓGICAS VERBALES ARGUMENTATIVAS INGLESAS (VAC) EN LAS MANIOBRAS DE REMOLQUE MARÍTIMO: DETECCIÓN Y REPRESENTACIÓN DE SUS PROPIEDADES SINTÁCTICAS Y SEMÁNTICAS

RESUMEN: En este artículo se investigan las construcciones fraseológicas verbales inglesas inscritas en la estructura argumentativa del verbo (VAC) usadas en las expresiones sobre las maniobras de remolque maritimo con la marca semántica [+MOVIMIENTO]. El objetivo de este estudio, basado en un corpus ad hoc especializado, es identificar las construcciones fraseológicas verbales y representar sus rasgos sintácticos y semánticos con el objetivo de sentar las bases para la elaboración de un recurso término-ontológico que pueda contribuir a la mejora de las competencias terminológicas y contextuales de los profesionales de la lengua y del ámbito marítimo. El método de indagación del corpus ha sido semasiológico y onomasiológico a través del establecimiento de unas pautas para la exploración del patrón verbo + argumento y del uso de la clase semántica de verbos push/pullde Levin (1993). El resultado es la aportación de un inventario de fraseologia verbal inglesa sobre las operaciones del remolcador marítimo.

PALABRAS CLAVES: construccione verbales argumentativas en inglés; fraseología especializada del discurso técnico-marítimo; descripción sintácticosemántica; recursos término-ontológicos basados en corpus; terminología en inglés sobre el remolcador maritimo.

SUMARIO: 1. Introducción. 2. Estudios previos. 3. El evento Tow. 4. Datos del corpus. 5. Método. 6. Análisis. 7. Resultados. 8. Conclusión.
ENGLISH PHRASEOLOGICAL VERB ARGUMENT CONSTRUCTIONS (VAC) IN THE MARITIME TOWAGE OPERATIONS: DETECTION AND REPRESENTATION OF THEIR SYNTACTIC AND SEMANTIC PROPERTIES

ABSTRACT: This article explores the English phraseological verb constructions found within the verb argument construction (VAC) in the expressions used during the maritime towage operations which present the semantic feature [+MOVEMENT]. The purpose of this study, which is based on a specialized ad hoc corpus, is to identify the verb phraseological constructions and represent their syntactic and semantic properties. This will help to lay the foundations for the building of a term-ontological tool that may contribute to the enhancement of the terminological and contextual competences of the language professionals and of maritime language users. The corpus enquiry method has been both semasiological and onomasiological through the establishment of a step-by-step procedure for exploring the verb + argument patterns and the application of the push/pull semantic verb class by Levin (1993). The result of this study is the contribution of an inventory of English verb phraseology for the maritime towage operations.

KEY WORDS: English verb argumentative construction; specialized phraseology in the maritime technical discourse; syntactic-semantic description; corpus-based term-ontological tools; English terminology on maritime towage.

SUMMARY: 1 . Introduction. 2. Previous studies. 3. The Tow event. 4. Corpus data. 5. Method. 6. Analysis. 7. Results. 8. Conclusion.
LES CONSTRUCTIONS PHRASÉOLOGIOUES VERBALES ARGUMENTATIVES ANGLAISES (VAC) LORS DES MANOEUVRES DE REMORQUAGE MARITIME : DÉTECTION ET REPRÉSENTATION DE SES PROPRIÉTÉS SYNTAXIQUES ET SÉMANTIQUES

RÉSUMÉ : Cet article examine les constructions phraséologiques verbales anglaises inscrites dans la structure argumentative du verbe (VAC) dans les termes utilisés dans les manœuvres de remorquage maritime avec la marque séman tique [+MOUVEMENT]. L'objectif de cette étude, basée sur un corpus ad hoc spécialisé, est les identifier les constructions phraséologiques verbales et représenter leurs traits syntaxiques et sémantiques dans le but de jeter les bases de l'élaboration d'une ressource terme-ontologique pour des professionnels de la langue et le domaine maritime que contribuant à l'amélioration de ses compétences terminologique et contextuelles. La méthode d'enquête du corpus a été hebdomadaire et onomasiologique par l'établissement de lignes directrices pour l'exploration du motif verbe argument et de l'utilisation de la classe sémantique des verbes push/pull de Levin (1993). Le résultat est la fourniture d'un inventaire de phraséologie verbale en anglais sur les opérations du remorquage maritime.

MOTS CLÉS : constructions phraséologiques verbales anglaises ; phraséologie spécialisée du discours technico-maritime ; description syntaxiques et sémantiques ; ressources terme-ontologiques basées sur corpus ; terminologie en anglais sur le remorquage maritime.

SOMMAIRE : 1 . Introduction. 2. Études préalables. 3. L'événement Tow. 4. Le corpus de données. 5. Méthode. 6. Analyse. 7. Résultats. 8. Conclusion.
Fecha de Recepción Fecha de Revisión Fecha de Aceptación Fecha de Publicación 
Las construcciones fraseológicas verbales argumentativas
inglesas (VAC) en las maniobras de remolque maritimo:
detección y representación de sus propiedades sintácticas y
semánticas ${ }^{1}$

MARÍA-ARACELI LOSEY-LEÓN

\section{INTRODUCCIÓN}

Las unidades terminológicas verbales en las operaciones técnico-marítimas suelen ser abundantes en textos escritos y orales a causa de su vínculo discursivo con el transporte y el desplazamiento caracteristicos de este entorno. La marca semántica [+MOVIMIENTO] se aprecia directamente en verbos como navigate, turn, roll, sway, surge y move astern; sin embargo, se observa que no siempre está implícita en el verbo. A veces, la estructura argumentativa del verbo es la que provee de marcas semánticas para expresar movimiento, a través de una construcción dotada de un complemento, ya sea un sintagma nominal -de aquí en adelante, NP-, un sintagma preposicional -PP- o un sintagma adverbial -ADP-. Este es el caso de give a pull o push ahead. En este capítulo investigamos la fraseología inglesa que consta de verbo y argumento, concretamente abordaremos las construcciones $[\mathrm{V}+(\mathrm{NP})+(\mathrm{PP})+(\mathrm{ADP})]$ y $[\mathrm{V}+(\mathrm{PP})+(\mathrm{ADP})+(\mathrm{NP})]$ que se usan en las maniobras que implican movimiento del buque remolcador y del buque remolcado pues no se han encontrado estudios previos sobre esta parcela específica.

Los usuarios de la lengua inglesa especializada del ámbito marítimo han de manejar la terminología procedente de una multiplicidad de dominios y subdominios en los que se ramifica el conocimiento especializado de esta materia. El vocabulario de la industria maritima cuenta con el legado terminológico de las ciencias y técnicas de la navegación, de la ingeniería, del derecho, de la administración y de la medicina, al tiempo que es partícipe de la constante incorporación de neologismos, en particular los generados en la ciencia y en la tecnología. El transporte maritimo es una actividad especializada en la que se combinan los conocimientos técnicos y los jurídico-comerciales. Se desenvuelve en entornos con un denso flujo de transacciones marítimo-comerciales, pero también en aquellos con una intensa actividad técnico-maritima, tanto en los puertos como en las plataformas marinas y en las estaciones de tierra. La comunicación entre todos los agentes que intervienen es crucial y, en todo caso, ha de primar el entendimiento sobre la base de premisas claras e identificables para las partes implicadas, ya sea en la negociación de un acuerdo comercial o en la ejecución de una maniobra técnico-marítima. En el caso de esta última, adquiere especial relevancia el vínculo entre comunicación, riesgo y seguridad. El trabajo en el medio marino implica estar expuestos a accidentes por la propia

\footnotetext{
${ }^{1}$ El presente trabajo ha sido realizado en el seno del proyecto 'VIP: sistema integrado Voz-texto para IntérPretes' (ref. FFI2016-75831-P, MINECO).
} 
naturaleza del medio marino, por el manejo de maquinaria y utilería de gran tonelaje por parte de los tripulantes y de los equipos portuarios y por el error humano, entre ellos, una defectuosa comunicación en inglés.

La presente investigación aborda, desde una perspectiva lingüística, una de las maniobras más comprometidas con la eficacia comunicativa, la del remolque marítimo, en donde la coordinación de los movimientos entre el buque remolcado y el remolcador, el medio, el tiempo, el orden de las secuencias, la intervención de los agentes y la instrumentación son esenciales. El objetivo de esta investigación es el estudio de las construcciones fraseológicas verbales asociadas a las maniobras de remolque marítimo. Sus resultados podrán servir para sentar las bases de la creación de un recurso de fraseología verbal especializada basado en corpus, dirigido a los profesionales de las lenguas especializadas, al profesional del mar y a cualquier usuario interesado en este campo de la comunicación especializada. Para ello, se han explorado las unidades fraseológicas ${ }^{2}$ cuyo núcleo es el verbo identificadas a partir de su estructura sintáctica, asi como de su frecuenciay se han descrito las relaciones semánticas de los verbos implicados en la maniobra de remolque, tomando como referencia la construcción argumentativa del verbo.

La hipótesis que barajamos en este trabajo es que las unidades fraseológicas que expresan maniobras seguirán con mayor frecuencia un patrón morfosintáctico con partículas (preposiciones o adverbios), dada la importancia del concepto MOVIMIENTO, EMPUJE e INSTRUMENTACIÓN para realizar el evento ${ }^{3}$ remolque maritimo. Las preguntas de investigación planteadas son: 1) ¿Qué patrones de construcciones fraseológicas verbales en inglés del tipo argumentativas $[\mathrm{V}+(\mathrm{NP})+(\mathrm{PP})+(\mathrm{ADP})]$ y $[\mathrm{V}+(\mathrm{PP})+(\mathrm{ADP})+(\mathrm{NP})]$ se hallan en el corpus especializado Tug-Tow?; 2) ¿Cuál es el patrón más frecuente $\mathrm{y}$, por tanto, relevante, que caracteriza a la fraseología verbal del evento remolque maritimo?

Este estudio se estructura en ocho secciones. En la sección segunda se presentan los estudios previos sobre el tema; la sección tercera muestra las características del evento remolque maritimo. En la sección cuarta se describe el corpus Tug-Tow, el cual aporta los datos de la investigación, en la quinta sección se explica cuál es el método a partir del conjunto de datos arrojados por el corpus Tug-Tow y se establece el protocolo de estudio. La sección sexta muestra el desarrollo del análisis. En la sección séptima se exponen los resultados obtenidos y, en la octava, las conclusiones alcanzadas, junto a las dificultades y restricciones halladas, las acciones realizadas, al tiempo que se proponen nuevas vías de avance en la investigación.

\footnotetext{
2 El concepto de unidades fraseológicas que seguimos en nuestro estudio es, en términos de Wray (2000: 465), el de formulaic sequences, que denotan: "A sequence, continuous or discontinuous, of words or other meaning elements, which is, or appears to be, prefabricated: that is, stored and retrieved whole from memory at the time of use, rather than being subject to generation or analysis by the language grammar."

${ }^{3}$ En este estudio utilizamos evento desde el punto de vista semántico. En consonancia con la perspectiva de Leech (2004: 9), nos referimos a “'event' meanings or uses of verbs”.
} 


\section{ESTUDIOS PREVIOS}

Las construcciones léxico-gramaticales y su asociación a roles semánticos argumentativos han sido ampliamente abordadas por trabajos anteriores. En ellos, se ha examinado la relación entre la semántica del verbo y la estructura sintáctica argumentativa. Goldberg (1995) centró su atención en la extensión argumentativa verbal, concretamente en la denominada construcción de movimiento causado o "caused-motion construction" (CMC), representada genéricamente como [Subject [verb Object Directional]. Determinó que existen cinco tipos de construcciones en las que el movimiento queda explicado a través de la función de la partícula, al tiempo que dejaba patente el vínculo de la estructura sintáctica con la estructura a nivel semántico. Son varios los autores que, a partir de la línea iniciada por Goldberg, han continuado explorando y analizando las construcciones de verbo y argumento (Kay, 2005; Hwang y Palmer, 2015).

La estructura de la verb phrase 4 fue abordada por Palmer (1965) a propósito de su análisis de los verbos catenativos (172-214). Posteriormente, las construcciones verbales inglesas también han sido objeto de análisis bajo el prisma de Corpus Pattern Analysis de Hanks ${ }^{5}$ (2005), quien presenta un inventario de corte semántico de los verbos ingleses extraídos de corpus lingüísticos para mostrar la relación entre el significado y el uso del verbo. Proporciona información sobre la estructura de la oración y la categorización semántica del argumento verbal y propugna que el significado del verbo está vinculado al patrón en el que se halla, al que define como "a relationship between sets of collocates. A single word cannot be a pattern" (Hanks, 2005: 117). Puede deducirse, pues, que Hanks considera que un patrón, dado que lo equipara con una colocación, es una construcción o secuencia con un grado de fijación.

Es evidente que los estudios que se han inclinado hacia la descripción de la estructura argumentativa han debido indagar, necesariamente, en el estudio formal del verbo. Uno de los trabajos más conocidos es la clasificación de los verbos ingleses de Levin (1993). También cabe mencionar el estudio de las construcciones de verbo+partícula o verb-particle constructions (VPC) de Fraser (1986), entre otros. Clark y Clark (1979) proponen una clasificación de verbos sobre movimiento causado por un vehículo, a los que denominaron instrument verbs (verbos instrumentales). En 2000, Kipper et al. realizan un estudio para proveer de información sintáctica y semántica al

\footnotetext{
${ }^{4} \mathrm{Su}$ equivalente en español es sintagma verbal.

${ }^{5}$ Las características de CPA pueden consultarse en https://nlp.fi.muni.cz/projects/cpa/_Fecha de consulta: 12/02/2020). Este enfoque ha derivado en dos proyectos individuales en los que se han desarrollado un diccionario sobre los patrones de los verbos en inglés, The Pattern Dictionary of English Verbs (PDEV) que contiene alrededor de 1.700 verbos (Hanks, 2020) y disponible en www.pdev.org.uk (Fecha de consulta: 10/05/2020) y un diccionario sobre los patrones de los verbos en español, Pattern Dictionary of Spanish Verbs (PDSV), con 300 verbos. Este recurso forma parte de un proyecto liderado por Irene Renau, se puede consultar en www.verbario.com (Fecha de consulta: 10/05/2020) y está descrito en Baisa et al. (2016).
} 
lexicón verbal de WordNet $^{6}$ (Miller, 1995; Princeton University, 2010). También en el entorno electrónico, encontramos recursos léxicos como VerbNet ${ }^{7}$ (Kipper Schuler, 2005; Palmer et al., 2005), que se basa, fundamentalmente, en la clasificación sintáctico-semántica de los verbos ingleses de Levin (1993). VerbNet no recoge verbos de dominios especializados como tow (remolcar) pero sí otros que evocan semánticamente la acción básica o primaria de remolcar, como son press (presionar) o push (empujar). Otra de las bases de datos léxica con información de construcciones verbales es ProBank (Kingsbury y Palmer, 2002). Compuesta por un corpus de textos anotados con roles semánticos, indica los marcos semánticos básicos de las relaciones predicado-argumento. Contempla el marco del verbo tow y proporciona el argumento out to sea, que contextualiza a través del ejemplo que figura en el corpus del recurso, a saber: "Out in the harbour, preparations are being made to attach cables to the tanker so that it can be floated and towed out to the sea". ${ }^{8} \mathrm{El}$ rol semántico es "(try to) cause motion, cause motion", es decir, (intentar) causar movimiento, causar movimiento. La información sintáctica y la información semántica del ejemplo anterior es, como se puede observar, ciertamente restrictiva pues no contempla otra representación del evento verbal tow como, por ejemplo, la instrumentalización del evento o la acción específica que conlleva el remolque.

Actualmente, se puede acceder tanto a VerbNet como a PropBank desde el repositorio Unified Verb Index ${ }^{9}$, que tiene registrados 9344 verbos ingleses y en el que se alojan los enlaces a sitios web sobre proyectos de procesamiento de lenguaje natural, como es el caso de OntoNotes ${ }^{10}$, SynSemClass Lexicon $^{11}$ y FrameNet ${ }^{12}$. Precisamente, este último se ha constituido como uno de los recursos más completos en la representación léxico-semántica. FrameNet es una base de datos que ofrece una amplia y detallada descripción de la combinatoria del léxico a través de los marcos semánticos (Fillmore, 1982). Si bien no recoge el verbo tow sí analiza verbos periféricos como

\footnotetext{
${ }^{6}$ Durante la consulta realizada en 2020 se ha comprobado que WordNet recoge el verbo tow como "tug (tow (a vessel) with a tug)". Disponible en http://wordnetweb.princeton. edu $/$ perl $/$ webwn?o2 $=\& \circ 0=1 \& 08=1 \& 01=1 \& 07=\& 05=\& 09=\& 06=\& 03=\& 04=\& s=$ tow $\& i=6 \& h=01101$ 000\#c (Fecha de consulta: 20/03/2020).

${ }^{7}$ Es una base de datos léxica basada en las clases de verbos ingleses de Levin (1993) a la que también se han ido incorporando las clases y la categorización sintáctico-semántica de verbos propuestas por Korhonen y Briscoe (2004), consistente en 57 clases nuevas de tipos verbales que se añaden a las de Levin. Otra extensión de la clasificación verbal está recogida en Kipper et al. (2008).

${ }_{8}$ Nuestra cursiva. Se puede acceder a la descripción en http://verbs.colorado.edu/propbank /framesets-english-aliases/tow.html (Fecha de consulta: 15/01/2020).

${ }_{9}$ Disponible en https://uvi.colorado.edu/ (Fecha de consulta: 15/01/2020).

10 Para más información sobre el proyecto se puede consultar el sitio web particular: http://clear.colorado.edu/compsem/index.php?page=lexicalresources\&sub=ontonotes (Fecha de consulta: 15/01/2020).

${ }^{11}$ Este proyecto, que se desarrolla en el Institute of Formal and Applied Linguistics de Charles University, se basa en sinonimia contextual y valencia de verbos. Para una información más completa sobre el trabajo desarrollado puede consultarse el sitio web particular: https://ufal.mff.cuni.cz/synsemclass (Fecha de consulta: 06/03/2020).

$12 \mathrm{Su}$ sitio web particular dispone de información concreta sobre el proyecto: https://framenet.icsi.berkeley.edu/fndrupal/ (Fecha de consulta: 16/01/2020).
} 
pull, push, haul, drag, jerk, press, roll, shove, thrust y tug que comparten el marco semántico <Cause_motion $>13$

En esta investigación, la clasificación de los verbos de Levin (1993) se torna significativa por ser punto de partida para el análisis de los verbos que clasifica semánticamente como verbos que ejercen fuerza o presión, tal es el caso de empujar/tirar (push/pull) y que son centrales en nuestro objeto de estudio. Si bien Levin en su clasificación de 1993 no incluye el verbo to tow (remolcar), sí presenta a los verbos draw, heave, jerk, press, pull, push, shove, thrust, tug, yank (Levin, 1993: 137), sobre los que considera que, aunque son verbos de movimiento no direccional en si, pueden ir acompañados de una frase con un argumento que exprese direccionalidad. Este listado de verbos es prácticamente igual que el de FrameNet.

Al hilo de esta cuestión, se ha podido detectar que, en el caso del verbo tow, se han dado realizaciones en las que este verbo sí puede indicar direccionalidad. Observemos los siguientes ejemplos:

(1) The tug towed the vessel. (E1 remolcador remolcó al buque).

(2) The tug towed the vessel to its position. (El remolcador remolcó al buque hasta su posición).

En ambas oraciones se ilustra la transitividad del verbo tow pero, mientras que en (1) no implica direccionalidad, en (2) sí, ayudado por la intervención de la partícula to. Además, añade un dato sobre una localización, position, reforzando la capacidad informativa y relevante del mensaje al indicar que el buque realizó su tarea y la completó, es decir, lo trasladó hasta la posición prevista final. En (1), en cambio, no se contempla. Por tanto, la señalización de direccionalidad o no, parece depender de la construcción fraseológica argumentativa verbal [V+NP+PP].

Más adelante, en un trabajo posterior, en esta ocasión de Levin y Rappaport Hovav (1995: 265-266), se recopilaron un conjunto de 125 verbos bajo la categoria de Agentive verbs of manner of motion pero tampoco se halla tow, aunque sí figuran verbos como roll (balancear), término aplicable a uno de los movimientos del buque sobre su eje longitudinal.

En cuanto a las investigaciones anteriores sobre los recursos terminológicos específicamente orientados al remolcador maritimo no se han podido localizar y se percibe una carencia de recursos lingüisticos que sirvan de soporte de consulta y de aprendizaje.

\section{El EVENTO TOW}

En este apartado se intentará definir y contextualizar la actividad de remolque maritimo. Los remolcadores desarrollan su actividad en distintos

13 La descripción completa de este marco semántico se puede localizar en https:// framenet2.icsi.berkeley.edu/fnReports/data/frameIndex.xml?frame=Cause_motion (Fecha de consulta: 16/01/2020). 
entornos marinos, en el litoral, en las áreas portuarias, en ríos y aguas interiores (vias navegables) y en mar abierto. Su función es asistir a los buques en la realización de las maniobras de atraque y desatraque en puerto, así como realizar labores de remolque a buques desde alta mar con fines de rescate o de colaboración en el traslado y posicionamiento de plataformas. La forma en la que se gestiona su actuación es a través de un contrato, ${ }^{14}$ mediante el cual, el buque remolcador, a cambio de una tarifa, se compromete a realizar la maniobra necesaria para el desplazamiento de otro buque. Se distinguen dos modalidades, a saber, a) el remolque transporte: en él, salvo pacto expreso en contrario, la dirección de la maniobra corresponde al capitán del remolcador y b) el remolque maniobra: en este caso, el remolque tiene por objeto la asistencia del remolcador a la maniobra del remolcado; se entenderá que, salvo pacto en contrario, la dirección de la maniobra recae sobre el mando del buque remolcado.

El verbo central en torno al que se desarrolla la operación principal del remolcador es tow. Como antesala a un estudio detallado del mismo, se presentan, a continuación, los resultados de la consulta realizada en diccionario ingleses y españoles de distintas épocas para conocer su conceptualización primitiva y su evolución histórica. En el diccionario especializado Nautical Dictionary (Young, 1863), se define tow como "To draw a vessel along by means of a rope attached to another ship or boat" (421). Ilustra, pues, el sentido primitivo de remolcar, es decir, halar, arrastrar o tirar de un buque mediante un cabo atado a otro buque o embarcación. A Dictionary of Sea Terms (Ansted, 1920) lo define como "to draw a vessel along in the water"(...) y extiende su definición incorporando información sobre las modalidades de remolque: "It may be done either from banks, by horses or men, or by another vessel in the water, as a tug takes a ship in tow" (311). Diccionarios generales contemporáneos, como Cambridge Dictionary, lo definen como "to pull (a ship, barge, car, trailer, etc.) by a rope, chain or cable." Se puede deducir, pues, que la voz tow y su significado primitivo se han mantenido a lo largo de la historia.

En cuanto a su equivalente en español, el término remolcar ya aparecía en el Diccionario de Autoridades, tomo V (1737) como "Voz naut. que vale dar cabo a la embarcación, que por mui cargada, o por otra razón, no puede caminar: y así se le ayuda atándola a otra, para conducirla al Puerto, o sacarla afuera". ${ }^{15}$ Se puede observar que, en la definición del término español, no se utiliza un verbo de acción que indica directamente remolcar. En el caso del inglés, utiliza el verbo draw (arrastrar). No será hasta 1825 que se define remolcar con un verbo más preciso, el verbo tirar, quedando recogido así por Núñez de Taboada en el Diccionario de la Lengua Castellana: "llevar

\footnotetext{
${ }^{14}$ Según lo dispuesto en la Ley 14/2014, de 24 de julio, de Navegación Marítima (BOE núm. 180, de viernes, 25 de julio de 2014, pág. 59261). Recuperado de https://www.boe.es/boe/dias/2014/07/25/pdfs/BOE-A-2014-7877.pdf. (Fecha de consulta: 15/09/2019).

${ }^{15}$ Se puede consultar en http://web.frl.es/DA.html (Fecha de consulta: 22/01/2020). Según consta en la página web de la Real Academia Española, el recurso electrónico para acceder a la consulta de este diccionario ha sido elaborado por el Instituto de Investigación Rafael Lapesa y editado por la Real Academia Española.
} 
alguna embarcación u otra cosa sobre el agua, tirando de ella por medio de algún cabo."16 En diccionarios contemporáneos, como la edición en línea del Diccionario de la Real Academia de la Lengua Española, se define remolcar así: "Llevar una embarcación u otra cosa sobre el agua, tirando de ella por medio de un cabo o cuerda". ${ }^{17}$

Se puede colegir que, a partir de los datos proporcionados por las fuentes terminográficas consultadas, los rasgos semánticos básicos de la acción tow (remolcar) son [TIRAR DE]+[ALGO]+[UTILIZANDO ALGO]. Puede, además, representarse semánticamente de dos formas, en función del fin de la maniobra de remolque:

1) PULL_SOMETHING_USING SOMETHING: Si la acción de la maniobra de remolque es la de ayudar al buque a entrar o salir de puerto, dadas sus dimensiones o las particularidades del muelle, puerto o atracadero.

2) FORCE_SOMETHING_TO MOVE_USING AN INSTRUMENT, si la acción consiste en remolcar a un buque varado, embarrancado o sin medios de propulsión.

El proceso cognitivo de conceptualización del evento remolque marítimo, en su doble modalidad, de remolque-maniobra y de remolque-transporte, requiere la identificación del vehículo ${ }^{18}$ en el que se realiza la actividad, de los agentes que gestionan la instrumentalización del vehículo, del espacio en el que se gestiona (espacio maritimo) y de los instrumentos en los que se apoya para realizar la actividad. Además, requiere una gestión adecuada del tiempo, ya que la acción recae en unas pautas espaciotemporales y secuenciadas. Disponer de una información organizada y estructurada del orden en el que se producen los distintos eventos del remolque puede ser decisivo, por ejemplo, en actividades de traducción, a la hora de asignar las equivalencias de la lengua origen a la lengua meta y de interpretación, al transmitir con precisión la concatenación de las acciones que participan.

\section{DATOS DEL CORPUS}

El corpus de estudio, Tug-Tow, es una extensión del corpus TugCorp, que fue compilado para un estudio anterior sobre la producción automática de frases normalizadas sobre el remolque maritimo para las comunicaciones maritimas. Tug-Tow puede definirse como un corpus escrito electrónico, especializado en el dominio del buque remolcador, monolingüe (inglés) y etiquetado morfológicamente, sincrónico, no equilibrado (no contiene el

\footnotetext{
${ }^{16}$ La ruta de consulta de la fuente ha sido la edición electrónica del Nuevo Tesoro Lexicográfico de la Lengua Española. Recuperado de http://ntlle.rae.es/ntlle/SrvltGUIMenuNtlle? cmd=Lema\&sec=1.0.0.0.0. (Fecha de consulta: 21/01/2020).

${ }_{17}$ Se puede consultar en: https://dle.rae.es/remolcar (Fecha de consulta: 15/09/2019).

18 La Ley 14/2014, de 24 de julio, de Navegación Marítima, establece que un buque es un "vehículo destinado a la navegación, que cubre también situaciones estáticas transitorias, como es el buque en construcción, fondeado, varado o en desguace" (Boletín Oficial del Estado, núm. 180, del viernes 25 de julio de 2014, Preámbulo, Sec. I. Pág. 59194). Recuperado de https://www.boe.es/boe/dias/2014/07/25/pdfs/BOE-A-2014-7877.pdf. (Fecha de consulta: $15 / 09 / 2019$ ).
} 
mismo número de textos por tipo textual) y cerrado. Es un corpus variado porque incluye tipos de textos diversos, a saber, manuales técnicos, descripciones técnicas y normativa técnica. En cuanto al nivel discursivo, contiene textos altamente especializados dirigidos a usuarios expertos y a semi-expertos. Según la estratificación temática y textual del corpus, es compuesto y modular. Como se ha indicado, el corpus Tug-Tow es una extensión de un corpus anterior, pero funciona como un módulo independiente y contiene, como se indica en la tabla 1, 226.464 tokens (palabras) y 13.698 types (formas).

\begin{tabular}{|c|c|c|}
\hline Corpus & $\mathrm{N}^{\circ}$ de tokens (palabras) & $\mathrm{N}^{\circ}$ de types (formas) \\
\hline Tug-Tow & 226.464 & 13.698 \\
\hline TugCorp & 7.154 & 785 \\
\hline
\end{tabular}

Tabla 1: Tamaño del corpus Tug-Tow y del corpus TugCorp

(Fuente: elaboración propia)

El corpus Tug-Tow consta de 124 documentos y las fuentes documentales proceden de instituciones marítimas, de carácter técnico y normativo, así como de fuentes académicas. Según el área temática, el corpus contiene textos científico-técnicos y jurídico-administrativos. En cuanto a la modalidad discursiva, los textos pertenecen al tipo normativo -con la reglamentación de cómo ha de equiparse un buque remolcador, sus revisiones, su equipamiento-, al tipo instructivo, al que pertenecen los manuales técnicos en todas sus modalidades -ficha técnica, especificación técnica, folleto técnico, boletín- y al descriptivo, en el que se detalla en un informe lo acontecido durante la travesía. Los textos han sido cuidadosamente seleccionados pues algunas de estas publicaciones son monografias sobre el tipo de buque o tipo de informe, por lo que se han ido localizando manualmente, uno a uno, solo aquellos que versan exclusivamente sobre los remolcadores.

Además del corpus de estudio, utilizaremos como corpus de referencia, el corpus TransShip, compuesto por distintos tipos de géneros textuales sobre los buques mercantes en general. Puede definirse como corpus electrónico, especializado, bilingüe, comparable, bidireccional (inglés-español; español-inglés), no anotado, variado y con nivel de especialización 1-2. ${ }^{19}$ Tanto el corpus de estudio como el corpus de referencia han sido pre-procesados, lo que significa que se han convertido en .txt y se les ha aplicado un filtro que elimina los caracteres y el ruido irrelevantes. El corpus Tug-Tow se ha etiquetado para la segunda fase y se ha lematizado.

Antes de aplicar todo el método para el procesado y análisis de los datos, se ha realizado como paso previo un sondeo al corpus para establecer su representatividad. Para ello, se ha extraído una lista de palabras con el programa Antconc 3.5.0 (Anthony, 2017) del corpus Tug-Tow y se han comparado los resultados con la lista de palabras obtenida del corpus SMCP-EXT,

\footnotetext{
${ }^{19}$ En la investigación se han utilizado los siguientes indicadores para los distintos niveles de especialización del texto en función del tipo de usuario a quien va dirigido: nivel 1 (expertoexperto); nivel 2 (experto-semi-experto); nivel 3 (experto-lego).
} 
también de creación propia, que contiene fraseología normalizada para las comunicaciones maritimas. Los resultados desvelan que tug ocupa la primera posición como candidato a término, con una frecuencia de aparición de 2.447 veces, seguido de towing, con 2.371 veces; de vessel (2.004 veces) y de ship (1.654 veces). En la posición cuarta figura tow con un índice de 1356 veces; es decir, el objeto y la acción verbal objeto de estudio son los candidatos a término más frecuentes. El hecho de que vessel y ship aparezcan en posiciones destacadas puede deberse a dos razones: o bien son parte constituyente y base de la unidad terminológica polilexemática y variante de tug (towing vessel o towing ship), o bien actúan como unidades terminológicas monolexemáticas en posiciones de función de referencia anafórica o catafórica. Igualmente significativa es la aparición de master (975) en la posición séptima y con una relevancia justificada como agente al mando pues es el término asignado al capitán, tanto del buque remolcador como del buque remolcado. Si comparamos estos resultados con los obtenidos en el módulo de listado de palabras de AntConc 3.5.0 para el corpus SMCP-EXT, sobre frases normalizadas para las comunicaciones maritimas, el cual contempla un apartado exclusivo, aunque breve, sobre los remolcadores, se observa que tanto tug como towing no son términos centrales y quedan relegados a posiciones periféricas. La mayoría aparece en posiciones inferiores a las que figura en el corpus Tug-Tow. Tug figura en la posición 26 con una frecuencia de 27 veces, mientras que cable, que puede ser una unidad de medida o un elemento del equipamiento del remolcador, figura en la posición 35 con una frecuencia de 39 veces. Los resultados indican que el corpus Tug-Tow es relevante para el estudio.

Con la búsqueda de palabras claves en el corpus Tug-Tow también se constata qué candidatos a término pueden categorizarse como unidades terminológicas. Se ha realizado con la medida estadística de verosimilitud y con un valor $\mathrm{p}$ de $<0.05$. En las primeras 10 posiciones destacan por su grado de especificidad y, por tanto, por ser peculiares de este dominio, las unidades terminológicas, tug, towing, vessel, ship, tow, emergency, safety, line, todas centrales en el dominio del remolcador marítimo. A continuación, se diseñó una plantilla de validación de las unidades terminológicas obtenidas tanto monolexemáticas como plurilexemáticas, la cual se entregó a expertos en transporte marítimo y en servicios portuarios para su validación, que fue positiva.

\section{MÉTOdo}

La aportación de datos a la investigación viene de la mano del corpus de estudio ad hoc, Tug-Tow, diseñado y compilado especialmente para esta investigación. El método que se ha seguido para responder a las preguntas de la investigación es de naturaleza híbrida (estadístico y lingüístico) y se despliega en dos fases en las que se combina el enfoque onomasiológico (del concepto al término) y semasiológico (del término al concepto): 
1) Fase 1: Detección y extracción automática de las unidades fraseológicas plurilexemáticas en las que el verbo sea la base. Se ha utilizado el método estadístico (aplicación del principio estadístico de mayor frecuencia de aparición de la unidad fraseológica plurilexemática) y lingüístico. Los pasos detallados han sido:

a) Generación de listados de concordancias indagando de forma selectiva en la búsqueda de verbos del mismo grupo semántico de tow, como pueden ser pull, push, heave, draw, thrust. El grupo de verbos que han servido de referencia son los correspondientes a la categoria pull/push de Levin (1993: 137).

b) Extensión del segmento de las concordancias hacia el argumento del predicado que incluya objeto y sintagma preposicional en distintas posiciones, $[\mathrm{V}+(\mathrm{NP})+(\mathrm{PP})+(\mathrm{ADP})]$ y $[\mathrm{V}+(\mathrm{PP})+(\mathrm{ADP})+(\mathrm{NP})]$

c) Desarrollo y asociación de los rasgos semánticos a cada construcción fraseológica.

2) Fase 2: Ampliación de búsqueda y extracción de verbos de maniobras de remolque maritimo distintos a la categoria push/pull de Levin, como pueden ser verbos que denoten acciones colaterales, como couple o let go; creación de grupos semánticos en los que se examinará la relación del verbo con el agente y el argumento; generación de inventario de fraseología verbal inglesa del dominio especializado (véase anexo I).

\section{ANÁLISIS}

Los datos se han analizado de forma cuantitativa y cualitativa y se han procesado de forma automática utilizando los programas AntConc 3.5.0 (Anthony, 2017) y TermoStat 3.0 (Drouin, 2003). La selección de la segunda fase se ha realizado de forma manual. Se han abordado los datos ofrecidos por el corpus. A continuación, se presentan los datos obtenidos y el procedimiento de desarrollo del análisis en las dos fases previstas.

\subsection{Fase 1: Detección y EXTRacción automática de las unidades fRaseológicas PLURILEXEMÁTICAS EN LAS QUE EL VERBO ES LA BASE}

A partir de distintas secuencias de búsqueda, se han generado listados de concordancias en los que se ha indagado de forma selectiva en la búsqueda del verbo tow y de los verbos incluidos en la clasificación semántica de verbos pull/push de Levin (1993): pull, push, draw, heave, jerk, press, shove, thrust, tug y yank. A continuación, se extenderá el segmento de búsqueda de las concordancias para localizar e identificar los patrones de argumento del predicado comprendidos en los siguientes segmentos: $[\mathrm{V}+(\mathrm{NP})+(\mathrm{PP})+(\mathrm{ADP})]$ y $[\mathrm{V}+(\mathrm{PP})+(\mathrm{ADP})+(\mathrm{NP})]$. Después de su detección, se ha representado en una tabla la construcción fraseológica y se ha dotado a cada una de ellas de un patrón semántico. El perfil semántico de cada verbo es VERB_MOVE. 


\subsubsection{TOWING}

Se ha optado por comenzar por la forma towing porque no solo puede arrojar resultados en los que forme parte de unidades terminológicas sustantivas polilexemáticas sino de forma verbal progresiva o "non-finite". Después de aplicar el módulo de clusters o secuencias de palabras de Antconc 3.5.0 con los parámetros de dos a la izquierda y dos a la derecha de la palabra base towing, se obtuvo un total de 359 candidatos a unidades terminológicas polilexemáticas. Se pudo comprobar, pues, que existe un grueso importante de términos en los que towing funciona como colocado de una colocación nominal (towing arrangements, towing operations, towing equipment, towing hook, towing vessel, towing winch, towing pennant, towing line, towing gear, towing procedures, towing point, towing manual, entre otros). Al aplicar la opción de secuencias de cuatro palabras (véase figura 5), surgieron más muestras de construcciones fraseológicas que implican movimiento y direccionalidad. Por ejemplo, towing from the stern (remolque desde popa), towing from the bow, (remolque desde proa), asi como elementos del equipo: towing hook (gancho de remolque) y towing line (cabo de remolque). Utilizando el módulo de concordancias, se obtuvieron 2371 líneas de concordancia con el término clave towing. Como unidad terminológica verbal y como verbo transitivo, towing aporta datos desde la estructura [V+Obj] sobre el objeto al que causa el movimiento. Por ejemplo, en las combinaciones towing a barge, towing a dumb dredger.

En cuanto a la estructura fraseológica [V+ADP], se asocia, primordialmente, al grupo semántico de acción y direccionalidad de la misma. Así, se pueden mencionar towing aft y towing foreward, los cuales se ilustran en la siguiente frase extraida del corpus Tug-Tow: "For ASD tug towing foreward the yawing effect is much greater than towing aft."

\subsubsection{VERBO TO TOW}

\begin{tabular}{|c|c|c|c|}
\hline \multicolumn{3}{|c|}{ Iotal No. of Cluster Types } & \multirow{2}{*}{$\begin{array}{l}405 \\
\text { Cluster }\end{array}$} \\
\hline Rank & Freq & Range & \\
\hline 1 & 268 & 33 & tow line \\
\hline 2 & 59 & 8 & tow wire \\
\hline 3 & 51 & 10 & tow rope \\
\hline 4 & 33 & 5 & tow winch \\
\hline 5 & 30 & 16 & tow lines \\
\hline 6 & 30 & 10 & tow was \\
\hline 7 & 29 & 12 & tow and \\
\hline 8 & 26 & 12 & tow to \\
\hline 9 & 25 & 6 & tow ropes \\
\hline
\end{tabular}

Figura 1: Extracto de resultados obtenidos con el módulo de secuencias de 2 palabras usando como término de búsqueda tow (Fuente: elaboración con AntConc 3.5.0)

Los datos reflejan un índice elevado de frecuencia en primeras posiciones en las que figura, mayoritariamente, como unidad terminológica sustantiva y como colocativo. En primera posición, con la secuencia tow line (286), tow lines (30), tow winch (33), tow rope (51) y tow wire (59), de un total de 405 
formas (types). Otro de los datos obtenidos refleja una alternancia en las variaciones sobre los términos aplicados al equipo, pudiéndose encontrar tanto towing line (voz dirigida a destacar la propiedad del cabo) como tow line (voz centrada en el propósito o fin del cabo). Una búsqueda más precisa del verbo to tow devuelve una producción más abundante, con un total de 38 formas. El método de extracción utilizado ha consistido en realizar búsquedas de secuencias de 5 palabras en las que el término de búsqueda era tow.

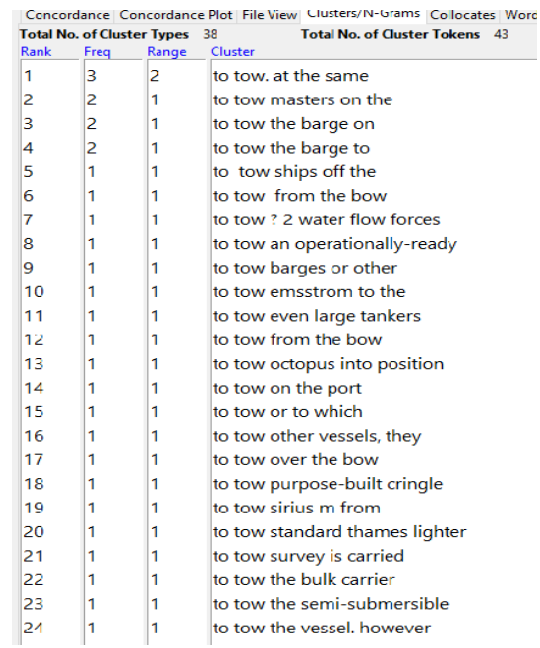

Figura 2: Extracto de resultados obtenidos con el módulo de secuencias de 5 palabras usando como término de búsqueda to tow (Fuente: elaboración con AntConc 3.5.0)

A continuación, en la tabla 2 se muestran las construcciones fraseológicas verbales detectadas ${ }^{20}$ y su descripción sintáctica y semántica:

\begin{tabular}{|c|c|c|}
\hline $\begin{array}{c}\text { Frase del corpus y } \\
\text { frecuencia de aparición }\end{array}$ & Rasgo semántico & $\begin{array}{c}\text { Construcción } \\
\text { fraseológica }\end{array}$ \\
\hline $\begin{array}{l}\text { tow from the stern (12) } \\
\text { tow from the bow (11) }\end{array}$ & TOW_MOVE_FROM DIRECTION & {$[\mathrm{V}+\mathrm{PP}]$} \\
\hline $\begin{array}{l}\text { tow a barge (2) } \\
\text { tow a dumb dredger (1) } \\
\text { tow other vessels (1) }\end{array}$ & TOW_MOVE_VEHICLE & {$[\mathrm{V}+\mathrm{NP}]$} \\
\hline tow ships off (1) & $\begin{array}{c}\text { TOW_MOVE_VEHICLE_DIREC- } \\
\text { TION_ } \\
\text { SEPARATION } \\
\end{array}$ & {$[\mathrm{V}+\mathrm{NP}+\mathrm{ADP}]$} \\
\hline $\begin{array}{c}\text { tow aft }(2) \\
\text { tow foreward }(2)\end{array}$ & TOW_MOVE_DIRECTION & {$[\mathrm{V}+\mathrm{ADP}]$} \\
\hline tow the barge to (2) & $\begin{array}{c}\text { TOW_MOVE_VEHICLE_DIREC- } \\
\text { TION } \\
\end{array}$ & {$[\mathrm{V}+\mathrm{NP}+\mathrm{ADP}]$} \\
\hline $\begin{array}{l}\text { tow alongside (6) } \\
\text { tow into position (5) }\end{array}$ & TOW_MOVE_POSITION & {$[\mathrm{V}+\mathrm{ADP}]$} \\
\hline tow on the port quarter (1) & TOW_MOVE_LOCATION & {$[\mathrm{V}+\mathrm{PP}]$} \\
\hline
\end{tabular}

Tabla 2: Caracterización sintáctica y semántica de las construcciones fraseológicas de base verbal tow (Fuente: elaboración propia)

${ }^{20}$ La mayoría de las construcciones del sintagma verbal están constituidas por un phrasal verb, es decir, por una construcción verbo+partícula (preposición o adverbio). En esta clase se incluyen las siguientes combinaciones: verbo+preposición, verbo+adverbio, verbo+adverbio+preposición. 
Los rasgos semánticos TOW_MOVE_VEHICLE_DIRECTION_ SEPARATION y TOW_MOVE_VEHICLE_SEPARATION comparten la misma construcción sintáctica. Del mismo modo, TOW_MOVE_DIRECTION y TOW_MOVE_POSITION siguen el mismo patrón sintáctico, [V+ADP]. La construcción sintáctica $[\mathrm{V}+\mathrm{PP}[\mathrm{P}+\mathrm{NP}]]$ se utiliza para expresar los significados TOW_MOVE_LOCATION y TOW_MOVE_FROM DIRECTION. Por tanto, el perfil semántico del verbo tow, TOW_MOVE está vinculado a construcciones fraseológicas compartidas de forma interna.

La interfaz sintáctica-semántica del evento remolque maritimo revela que, posiblemente debido al carácter durativo del verbo tow ${ }^{21}$, el movimiento está vinculado a la dirección del mismo y a la posición final en la que se culmina el evento.

Seguidamente, se analizan los demás verbos de la categoría pull/push de Levin y se podrá comprobar si comparten los rasgos sintácticos y semánticos.

\subsubsection{VERBO TO PULL 22}

La búsqueda del verbo pull ofrece 412 líneas de concordancias en las que se despliegan unidades terminológicas sustantivas y verbales. La tabla 3 recoge todas las construcciones fraseológicas verbales identificadas en su procesamiento:

\begin{tabular}{|c|c|c|}
\hline $\begin{array}{c}\text { Frase del corpus } \\
\text { y frecuencia de aparición }\end{array}$ & Rasgo semántico & $\begin{array}{l}\text { Construcción } \\
\text { fraseológica }\end{array}$ \\
\hline $\begin{array}{l}\text { to pull astern (8) } \\
\text { to pull ahead (3) }\end{array}$ & PULL_MOVE_DIRECTION & {$[\mathrm{V}+\mathrm{ADP}]$} \\
\hline to pull down (1) & PULL_MOVE_LOCATION & {$[\mathrm{V}+\mathrm{ADP}]$} \\
\hline to pull a ship (2) & PULL_MOVE_VEHICLE & {$[\mathrm{V}+\mathrm{NP}]$} \\
\hline $\begin{array}{l}\text { to pull the barge away (1) } \\
\text { to pull the tug laterally (1) } \\
\text { to pull the workboat along } \\
\text { (1) }\end{array}$ & $\begin{array}{l}\text { PULL_MOVE_VEHICLE_ } \\
\text { DIRECTION }\end{array}$ & {$[\mathrm{V}+\mathrm{NP}+\mathrm{ADP}]$} \\
\hline to pull the stern away (1) & $\begin{array}{l}\text { PULL_MOVE_VEHICLE PART_DI- } \\
\text { RECTION }\end{array}$ & [V+NP+ADP] \\
\hline to pull full (1) & PULL_MOVE_POWER DEGREE & [V+ADP] \\
\hline $\begin{array}{c}\text { to pull against the fixed end } \\
\text { of the brake (2) }\end{array}$ & PULL_MOVE_DIRECTION_OBJECT & {$[\mathrm{V}+\mathrm{PP}+\mathrm{PP}]$} \\
\hline $\begin{array}{c}\text { to be pulled over by its tow } \\
\text { line (1) }\end{array}$ & $\begin{array}{c}\text { (BE)PULL_MOVE_DIRECTION_ } \\
\text { INSTRUMENT }\end{array}$ & {$[\mathrm{V}+\mathrm{ADP}+\mathrm{PP}]$} \\
\hline
\end{tabular}

Tabla 3: Caracterización sintáctica y semántica de las construcciones fraseológicas de base verbal pull (Fuente: elaboración propia)

\footnotetext{
${ }^{21}$ Independientemente del hecho de que esta investigación se ha centrado en el movimiento y no en la naturaleza temporal del mismo, en los segmentos recuperados y analizados del corpus no se han localizado construcciones argumentativas de duración temporal propiamente dichas, a saber, ni las de carácter delimitativo (especifican los límites de la extensión) ni las de carácter cuantitativo (especifican la cantidad de tiempo invertida).

22 Por motivos de espacio, no se muestra una captura de pantalla como evidencia de la búsqueda realizada con el programa AntConc 3.5.0.
} 
El procesado también ha proporcionado muestras de unidades terminológicas polilexemáticas sustantivas como pulling tug y pushing tug, las cuales denotan las dos acciones principales del remolcador, halar y empujar, respectivamente.

\subsubsection{VERBO TO PUSH ${ }^{23}$}

En el caso del verbo push, el número de líneas de concordancias es inferior al de los verbos anteriores. Del mismo modo, se detecta un indice inferior de sustantivos combinados con el lema push. No obstante, como dato revelador conviene mencionar que el verbo push no solo comparte los rasgos semánticos con los demás verbos de la categoría push/pull de Levin (1993), sino que, además, comparte la construcción fraseológica. En la tabla 4 se detalla la coincidencia de la construcción:

\begin{tabular}{|c|c|c|}
\hline $\begin{array}{c}\text { Frase del corpus } \\
\mathrm{y} \text { frecuencia de aparición }\end{array}$ & Rasgo semántico & $\begin{array}{l}\text { Construcción } \\
\text { fraseológica }\end{array}$ \\
\hline $\begin{array}{l}\text { push the tug (5) } \\
\text { push the ship (1) } \\
\text { push a barge (3) }\end{array}$ & PUSH_MOVE_VEHICLE & {$[\mathrm{V}+\mathrm{NP}]$} \\
\hline push stern against the ship (1) & $\begin{array}{l}\text { PUSH_MOVE_VEHICLE PART_DIREC- } \\
\text { TION_OBJECT }\end{array}$ & {$[\mathrm{V}+\mathrm{NP}+\mathrm{ADP}+\mathrm{NP}]$} \\
\hline be pushed astern (1) & (BE) PUSH_MOVE_DIRECTION & {$[\mathrm{V}+\mathrm{ADP}]$} \\
\hline push the ship a bit (2) & PUSH_MOVE_VEHICLE_POWER DEGREE & {$[\mathrm{V}+\mathrm{NP}+\mathrm{ADP}]$} \\
\hline push the messenger line back & PUSH_MOVE_OBJECT_DIRECTION & {$[\mathrm{V}+\mathrm{NP}+\mathrm{ADP}]$} \\
\hline push out the rope (1) & PUSH_MOVE_DIRECTION_OBJECT & {$[\mathrm{V}+\mathrm{PP}+\mathrm{NP}]$} \\
\hline
\end{tabular}

Tabla 4: Caracterización sintáctica y semántica de las construcciones fraseológicas de base verbal push (Fuente: elaboración propia)

\subsubsection{VERBO TO DRAW}

Los resultados obtenidos a partir de las 67 líneas de concordancia revelan que son muy escasas las apariciones de su forma verbal. Se detectaron las siguientes:

\begin{tabular}{|c|c|c|}
\hline $\begin{array}{c}\text { Frase del corpus } \\
\text { y frecuencia de aparición }\end{array}$ & Rasgo semántico & $\begin{array}{c}\text { Construcción } \\
\text { fraseológica }\end{array}$ \\
\hline To draw ahead $(1)$ & DRAW_MOVE_DIRECTION & {$[\mathrm{V}+\mathrm{ADP}]$} \\
\hline To draw the tug toward $(1)$ & DRAW_MOVE_VEHICLE_DIRECTION & {$[\mathrm{V}+\mathrm{NP}+\mathrm{PP}]$} \\
\hline
\end{tabular}

Tabla 5: Caracterización sintáctica y semántica de las construcciones fraseológicas de base verbal draw (Fuente: elaboración propia)

Se constata, a raíz de los resultados obtenidos del corpus Tug-Tow, que este uso de draw se aleja de la categorización de Levin (1993) ya que la acción de arrastrar reflejada en el corpus no es provocada ni buscada por la maniobra del remolcador, sino que es un efecto colateral que puede acontecer durante la operación, ya sea causado por la fuerza del mar, por la fuerza propulsora de la hélice o por diferencias de tensión de los cabos de remolque.

23 Por motivos de espacio, no se muestra una captura de pantalla como evidencia de la búsqueda realizada con el programa AntConc 3.5.0. 


\subsubsection{VERBO TO HEAVE}

Los resultados muestran construcciones fraseológicas verbales más variadas y productivas a pesar de que el número es significativamente menor, si se compara con algunos de los verbos ya examinados.

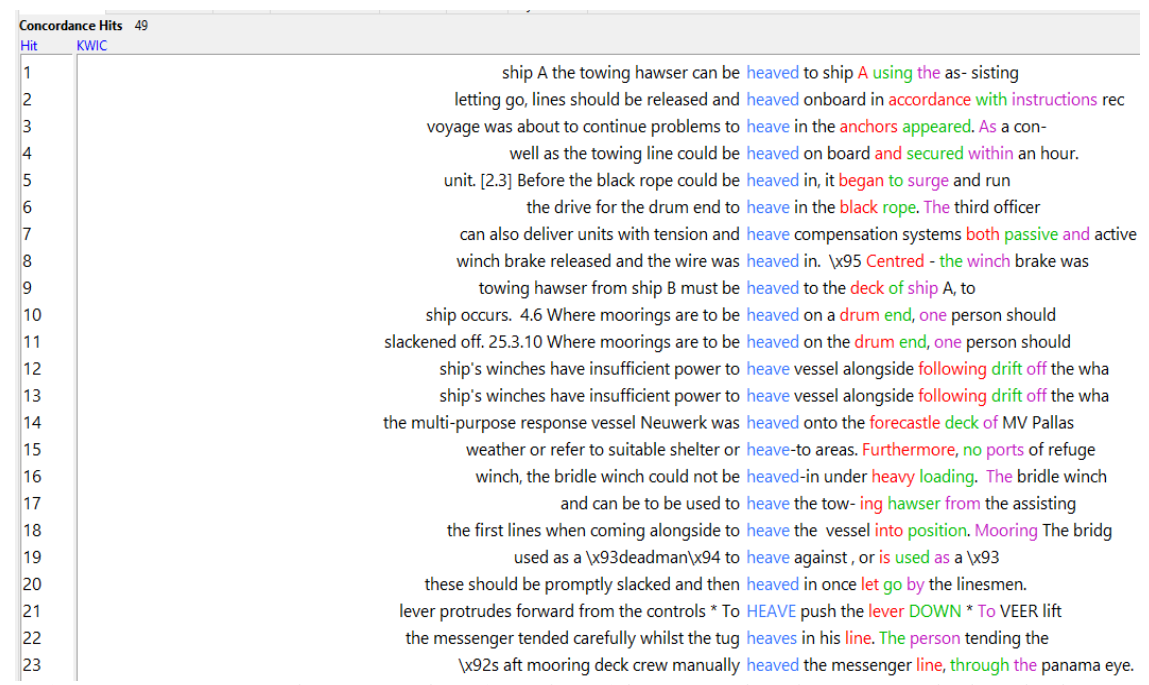

Figura 3: Extracto de concordancias obtenidas para las formas verbales de heave

(Fuente: elaboración con AntConc 3.5.0)

Además, la construcción verbo-partícula (verb-particle construction) es más frecuente que en los verbos anteriormente examinados. A continuación, se muestra la tabla de representación.

\begin{tabular}{|c|c|c|}
\hline $\begin{array}{c}\text { Frase del corpus } \\
\text { y frecuencia de aparición }\end{array}$ & Rasgo semántico & $\begin{array}{c}\text { Construcción } \\
\text { fraseologica }\end{array}$ \\
\hline $\begin{array}{c}\text { heave the vessel into position (1) } \\
\text { heave the vessel out of his } \\
\text { berth (1) }\end{array}$ & $\begin{array}{c}\text { HEAVE_VEHICLE_MOVE_ } \\
\text { LOCATION }\end{array}$ & [V+NP+PP] \\
\hline $\begin{array}{c}\text { heave in the messenger line (1) } \\
\text { heave in the black rope (1) } \\
\text { heave in the anchors (1) }\end{array}$ & HEAVE IN_INSTRUMENT \\
\hline $\begin{array}{c}\text { heave up the towline (1) } \\
\text { heave up the tow wire (1) }\end{array}$ & HEAVE UP_INSTRUMENT & [VPC+NP] \\
\hline heave the towing hawser (1) & HEAVE_INSTRUMENT & [V+NP] \\
\hline $\begin{array}{c}\text { heave the vessel off with beach } \\
\text { gear }(1)\end{array}$ & $\begin{array}{c}\text { HEAVE_VEHICLE_ } \\
\text { INSTRUMENT }\end{array}$ & [V+NP+ADP+PP] \\
\hline heave the vessel alongside (1) & $\begin{array}{c}\text { HEAVE_VEHICLE_MOVE_ } \\
\text { POSITION }\end{array}$ & [V+NP+ADP] \\
\hline
\end{tabular}

Tabla 6: Caracterización sintáctica y semántica de las construcciones fraseológicas de base verbal heave (Fuente: elaboración propia)

${ }^{24}$ VPC equivale a Verb+particle construction (construcción verbo+partícula). 
En ocasiones, aunque compartan el mismo grupo semántico hay ligeras variaciones en el patrón morfosintáctico. Heave $u p^{25}$ y heave in tienen la misma construcción fraseológica pero semánticamente son distintos. Heave in significa tirar o halar mientras que heave up es levar, acarrear hacia una posición superior en el eje vertical.

\subsubsection{VERBO TO PRESS}

Esta unidad terminológica no figura con la acepción de presión directa de las defensas del buque remolcador sobre el casco del buque remolcado para empujarlo, sino que los 14 registros en el corpus muestran la acción de presionar un mecanismo, por ejemplo, press the release button (pulse el botón de desenganche). Por este motivo no se procede a categorizarlo como un verbo generador de fraseología sobre el movimiento desencadenado por el evento remolque marítimo en sí.

\subsubsection{VERBO TO JERK}

El módulo de concordancias no devuelve muestras del verbo, pero sí despliega 16 registros de la forma sustantiva para hacer referencia a un tirón o sacudida del cabo de remolque. Por consiguiente, se puede considerar que este verbo adquiere la misma propiedad semántica en este contexto técnico que en los contextos ejemplificados por Levin (1993).

\subsubsection{VERBO TO SHOVE}

Este verbo implica "empujar bruscamente a algo o a alguien" pero no figura ningún registro por lo tanto su uso no parece ser relevante en este contexto, a juzgar por los resultados que arroja el corpus Tug-Tow.

\subsubsection{VERBO TO THRUST}

La lista de concordancias despliega un total de 205 ocurrencias, pero en ningún caso en forma verbal. Sí aparece el sustantivo thrust (empuje) y el propulsor de proa thruster. No se puede obtener una construcción fraseológica con argumento de predicado ya que no hay ocurrencias con la forma verbal.

\subsubsection{VERBO TO TUG}

Según Cambridge English Dictionary, el significado del verbo to tug es "to pull something sharply and strongly". Sin embargo, tras procesar el corpus no aparece ningún registro de esta voz. Por consiguiente, no es productivo

\footnotetext{
${ }^{25}$ A. Ansted (1986) en A Dictionary of Sea Terms, define heave up así: "to draw or pull up, as to heave up the anchor or a fishing net (127), es decir, "tirar para sí o levantar, como levar el ancla o una red de pesca" (traducción propia).
} 
en el contexto técnico aplicado a las maniobras de los remolcadores. Sí, en cambio, adquiere una posición central el sustantivo tug en este dominio, ya que es el término que, junto a towing vessel, hace referencia al remolcador. Existe una alternancia entre ambos términos si bien se constata una pequeña diferencia de uso. Towing vessel se utiliza en tipos de textos en los que el concepto primario es el de la construcción naval mientras que el término tug se tiende a utilizar con mayor frecuencia en contextos referentes al uso y a la función del buque. En el corpus Tug-Tow aparece un total de 3.223 veces.

\subsubsection{VERBO TO YANK}

Según los datos arrojados por el corpus, este verbo no se ha generado en el contexto técnico marítimo del remolcador.

\subsection{Fase 2: Detección de verbos implicados en las maniobras que no están CATEGORIZADOS EN LA CLASE DE LEVIN PARA COMPLETAR LA REPRESENTACIÓN DE LAS CONSTRUCCIONES FRASEOLÓGICAS VERBO +ARGUMENTO EN EL DOMINIO DE LAS MANIOBRAS DEL BUQUE REMOLCADOR}

Se realizó una validación de las unidades terminológicas con TermoStat 3.0 (Drouin, 2003), el cual proporciona el cálculo de especificidad del término aplicando el indice de variabilidad de las formas. Se extrajeron los verbos y los argumentos con el objeto de crear grupos semánticos. Además, se examinó la relación de cada verbo con el agente y con el argumento para acceder a la estructura conceptual del dominio del remolcador marítimo. Esto facilitará, en investigaciones futuras, elaborar un inventario de relaciones conceptuales.

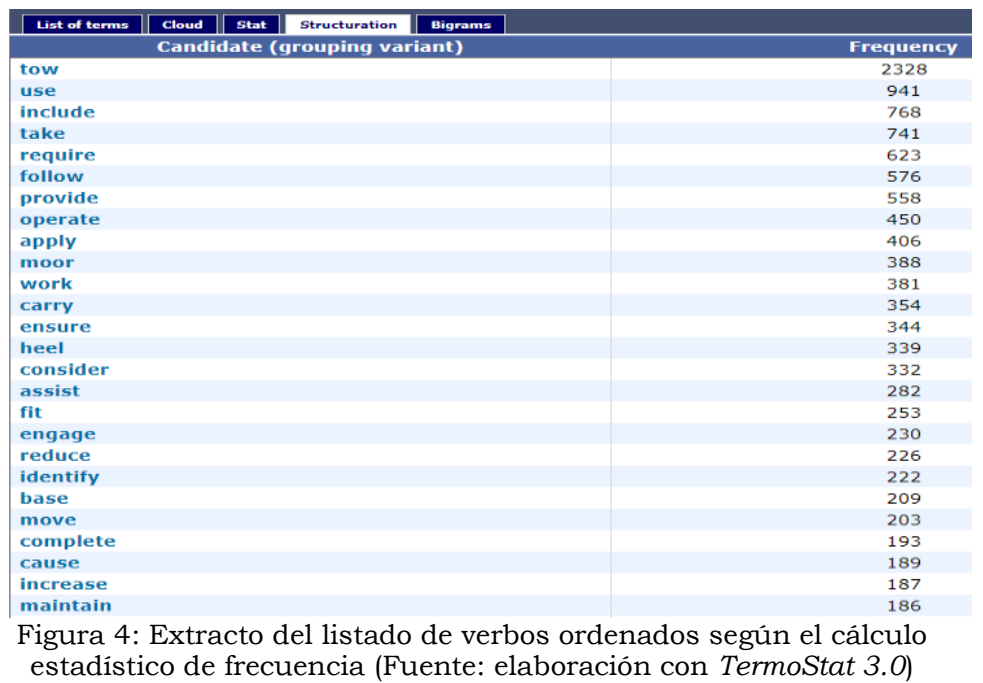


El procesado con TermoStat 3.0 (véase figura 4) revela la existencia de un total de 860 verbos diferentes y de una frecuencia de 63.617. La técnica que utilizamos para detectar los verbos que quedaban por localizar es la siguiente. En primer lugar, separamos los verbos de la categoría pull/push que ya habian sido analizados en la fase $1 \mathrm{y}$, en segundo lugar, teniendo en cuenta que el corpus contiene tipos de textos variados, tanto técnicos como normativos, hemos extendido la secuencia de sondeo a líneas de concordancias. Se han tenido en cuenta tanto verbos de carácter especializado como verbos semi-especializados o de tipo general (make, take, have, etc.) ya que también pueden formar combinaciones argumentativas relevantes. Esta parte de la $2^{\mathrm{a}}$ fase ha sido desarrollada manualmente. La nueva lista está compuesta por los verbos: use, include, take, make, require, operate, apply, carry, moor, give, ensure, heel, assist, fit, reduce, move, engage, go, increase, complete, keep, hold, maintain, pull, break, pass, secure y set.

TermoStat 3.0, como se ilustra en la figura 5, también nos permite obtener el índice de especificidad de los verbos del dominio, de manera que "tow" encabeza la lista con un índice de 181.78, seguido de "moor" (73.26), "manoeuvre" (40.79), “capsize" (40.96), "berth" (39.2) y “operate” (38.89). Se puede apreciar en la columna variants cuáles son las variantes en las que aparece cada verbo en el corpus.



Figura 5: Extracto de los 10 primeros verbos obtenidos y ordenados de mayor a menor según el índice de especificidad (Fuente: elaboración con Termostat 3.0)

La segunda parte de esta fase ha consistido en la designación y agrupación de todos los verbos constituyentes de las construcciones fraseológicas argumentativas inglesas (VAC) en grupos semánticos que se ilustran en la tabla 7 . 


\begin{tabular}{|c|c|}
\hline Grupos semánticos & Verbos ingleses \\
\hline empujar, halar, hacer presión & tow, pull, push, haul, hold, escort, guide \\
\hline accionar sobre un mecanismo o equipo & $\begin{array}{c}\text { release, reinforce, install, use, connect, keep, ad- } \\
\text { just, wind up, wind off, set, move, separate, hook, } \\
\text { detach, break, apply, engage, disengage, couple } \\
\text { (up) }\end{array}$ \\
\hline $\begin{array}{l}\text { parar el movimiento, suspender la presión o redu- } \\
\text { cir la presión que causa el movimiento }\end{array}$ & stop, stand by, finish, reduce \\
\hline $\begin{array}{l}\text { soltar, lanzar y recibir un elemento de un lado a } \\
\text { otro }\end{array}$ & receive, pass, send, give, slack \\
\hline subir un objeto, bajar un objeto & raise, lower \\
\hline sujetar y mantener un objeto & hold on, make fast, fasten, maintain \\
\hline asir un objeto & take \\
\hline $\begin{array}{l}\text { verificar el correcto funcionamiento de un objeto, } \\
\text { instrumento, mecanismo, dispositivo u equipo }\end{array}$ & check, make sure, ensure, secure \\
\hline
\end{tabular}

Tabla 7: Grupos semánticos de los verbos constituyentes de las construcciones fraseológicas verbales argumentativas en el dominio del remolque marítimo (Fuente: elaboración propia)

Finalmente, se ha consignado la fraseología verbal cuyas caracteristicas sintácticas y semánticas se han descrito en un inventario (véase tabla 1, anexo I).

\section{Resultados}

Del análisis de los datos se puede colegir que se ha podido dar respuesta a las preguntas planteadas en este estudio. Se han extraído un total de 42 frases constituidas por verbo + argumento y propias del dominio de las maniobras del remolcador maritimo. Una muestra del inventario se recoge en el anexo I. En respuesta a las preguntas planteadas en la investigación, se muestran los siguientes resultados:

\section{1) ¿Qué patrones de construcciones fraseológicas de predicado (verbo+argumento) del tipo argumentativas [V+(NP)+(PP)+(ADP)] y $[\mathrm{V}+(\mathrm{PP})+(\mathrm{ADP})+(\mathrm{NP})]$ se hallan en el corpus especializado Tug-Tow?}

De acuerdo con los datos obtenidos, se han obtenido las siguientes construcciones fraseológicas en respuesta a la primera cuestión de investigación: $[\mathrm{V}+\mathrm{PP}], \quad[\mathrm{V}+\mathrm{NP}], \quad[\mathrm{V}+\mathrm{ADVP}], \quad[\mathrm{V}+\mathrm{NP}+\mathrm{ADVP}], \quad[\mathrm{V}+\mathrm{PP}+\mathrm{PP}], \quad[\mathrm{V}+\mathrm{NP}+\mathrm{ADVP}+\mathrm{NP}]$, $[\mathrm{V}+\mathrm{PP}+\mathrm{NP}],[\mathrm{V}+\mathrm{NP}+\mathrm{PP}],[\mathrm{VPC}+\mathrm{NP}],[\mathrm{VPC}+\mathrm{NP}+\mathrm{PP}]$.

Hay un total de 10 construcciones fraseológicas verbales distintas que caracterizan este dominio, de las cuales 9 contienen una partícula, ya sea preposición, adverbio o partícula parte del verbo (phrasal verb). Por consiguiente, el gran peso de la argumentación verbal recae en el uso combinatorio de partículas que dan mayor precisión a la acción de verbos de la clase (push/pull). Cabe mencionar que los dos patrones matrices iniciales de construcciones argumentativas se completan de la siguiente forma: $[\mathrm{V}+(\mathrm{NP})+(\mathrm{PP} / \mathrm{ADP})+(\mathrm{PP} / \mathrm{ADP})+(\mathrm{NP})] \mathrm{y}[\mathrm{V}+(\mathrm{PP} / \mathrm{ADP})+(\mathrm{PP} / \mathrm{ADP})+(\mathrm{NP})]$ 


\section{2) ¿Cuál es el patrón más frecuente y, por tanto, relevante, en la fra- seologia verbal del evento remolque maritimo en el corpus Tug-Tow?}

No se ha podido determinar con total certeza y de forma completamente automática si hay un patrón más frecuente; en cambio, en consonancia con los hallazgos en el primer interrogante de este estudio, se puede deducir que el patrón más frecuente es aquel que contiene una partícula preposicional o adverbial que, además de reforzar la acción verbal, la concluye, tanto en la direccionalidad, operando bien desde o hacia un punto, como en el movimiento hacia la posición final. Uno de los datos que lo corroboran es la frecuencia de aparición de las construcciones fraseológicas con partícula para expresar direccionalidad. Por ejemplo, to tow from the stern/remolcar desde la popa, con una frecuencia de aparición de 12 veces, to tow from the bow/remolcar desde la proa, de 11 veces y to pull astern/tirar hacia popa, registrada ocho veces. La direccionalidad es un argumento sustancial en el efecto de fuerza y de presión activa y pasiva del remolcador sobre el buque remolcado. No hay que olvidar que la interacción entre los dos buques entraña dos rutas de direccionalidad; por un lado, la orientación de la acción verbal subyacente al remolque (to tow on the port quarter/remolcar por la aleta de babor) $\mathrm{y}$, por otro lado, la orientación de los cabos y amarras durante el intercambio de estas, desde y hacia los buques implicados (to send the towing line to the tug/dar el cabo de remolque al remolcador). En cualquier caso, la direccionalidad es necesaria para culminar el resultado de la acción y no solo está determinada por el verbo sino por la construcción fraseológica verbal argumentativa de movimiento o, en términos de Goldberg (1995), caused-motion construction. En la tabla 1 del anexo I se ilustra el índice de especificidad de los verbos más activos en estas composiciones. De los verbos obtenidos en la fase uno, destacan: tow (46) ${ }^{26}$, con un indice de especificidad de 181.78 , pull (22) ${ }^{27}$ con un indice de especificidad de 20.56 y heave (10) ${ }^{28}$ con un indice de especificidad de 30.5. En cuanto al siguiente patrón más frecuente, se puede determinar que es la construcción sobre la instrumentalización. Por ejemplo, to lower towing line to the $t u g /$ arriar $^{29}$ el cabo de remolque hasta el remolcador, con un registro de cuatro veces o to use the towing line of the vessel/usar el cabo de remolque del buque, que aparece en seis ocasiones. ${ }^{30}$

Para ilustrar la implicación de la partícula y, por tanto, del argumento, en la acción verbal, se ha ido asociando cada construcción a grupos semánticos.

El rumbo de la investigación ha llevado a extraer, localizar y determinar más verbos con propiedades semánticas distintas a la de hacer

\footnotetext{
${ }^{26}$ Número de frecuencia (véase la subsección 6.1.2).

27 Número de frecuencia (véase la subsección 6.1.3).

${ }^{28}$ Número de frecuencia (véase la subsección 6.1.6).

${ }^{29} \mathrm{El}$ Vocabulario de Construcción Naval (español-inglés; inglés-español), de Rafael Crespo Rodríguez (1979: 18) define y proporciona el equivalente de "arriar" así: "Aflojar, soltar, bajar o largar un cabo sobre vuelta, en banda, etc. - To lower"

${ }^{30}$ Las construcciones verbales sobre instrumentalización se han hallado, en su mayoría, durante la segunda fase del estudio (véase tabla 7).
} 
presión/fuerza sobre algo para ponerlo en movimiento, que participan en las maniobras de remolque marítimo. En la tabla 7 se presentan los verbos clasificados por grupos semánticos relacionados con el manejo de los cabos y equipos para realizar la maniobra de acople y desacople del remolcador al buque remolcado. Finalmente, la forma towing ha arrojado resultados significativos dado que su índice de unidades terminológicas sustantivas polilexemáticas ha desvelado que su número es considerablemente superior al de las construcciones argumentativas verbales con el verbo tow.

En un estudio posterior se procederá con la caracterización sintácticosemántica de los demás verbos localizados en el dominio del remolcador maritimo que figuran en la tabla 7 para ampliar el inventario fraseológico.

\section{Conclusiones}

En este estudio se ha accedido a las construcciones semánticas a partir de la extracción semi-automática de los patrones sintácticos de los verbos alojados en el corpus sobre el evento tow/remolcar. Son varios los estudios que respaldan la tesis de que los verbos con las mismas propiedades semánticas pueden tener las mismas combinaciones de patrones sintácticos. Desde la presente investigación, basada en un corpus especializado creado al efecto, Tug-Tow, se realiza, precisamente, una contribución a este hilo argumentativo en el caso concreto de construcciones verbales argumentativas inglesas con los rasgos semánticos propios de las acciones derivadas de las maniobras del remolque maritimo. Se han utilizado como punto de partida los verbos pull/push categorizados por Levin (1993), bajo la premisa de que las propiedades semánticas de estos verbos pueden ser las mismas que las de los verbos implicados en las maniobras del remolque marítimo, dado que empujar y tirar son dos acciones claves en las mismas. Se ha comprobado que no todos los verbos de esta categoría son relevantes o productivos en el remolque maritimo, a saber, jerk, press, shove, thrust, tug y yank. Por el contrario, si se han revelado significativamente productivos los demás verbos de su clase, objeto de estudio, en el ámbito técnico de la fraseología clave del remolcador marítimo y, por ello, componen parte importante del inventario de construcciones fraseológicas finales. Se ha procedido a ampliar el espectro de verbos prototípicos del dominio y se han representado sus correspondientes patrones sintácticos y semánticos. Se ha propuesto una clasificación de grupos semánticos del conjunto de los verbos de la clase push/pull de Levin (1993) más los que se han añadido como resultado de este estudio. Como producto y aportación de la presente investigación, un extracto del inventario de fraseología verbal argumentativa obtenida se presenta en el anexo I, con el indice de especificidad obtenido del programa TermoStat 3.0 (Drouin, 2003).

A continuación, en la figura 6, se representa la información sintácticosemántica de las construcciones fraseológicas en las que el verbo base es tow. En ella se vislumbra el agente productor, un agente receptor, un evento, un medio, un instrumento, una dirección y una localización. 


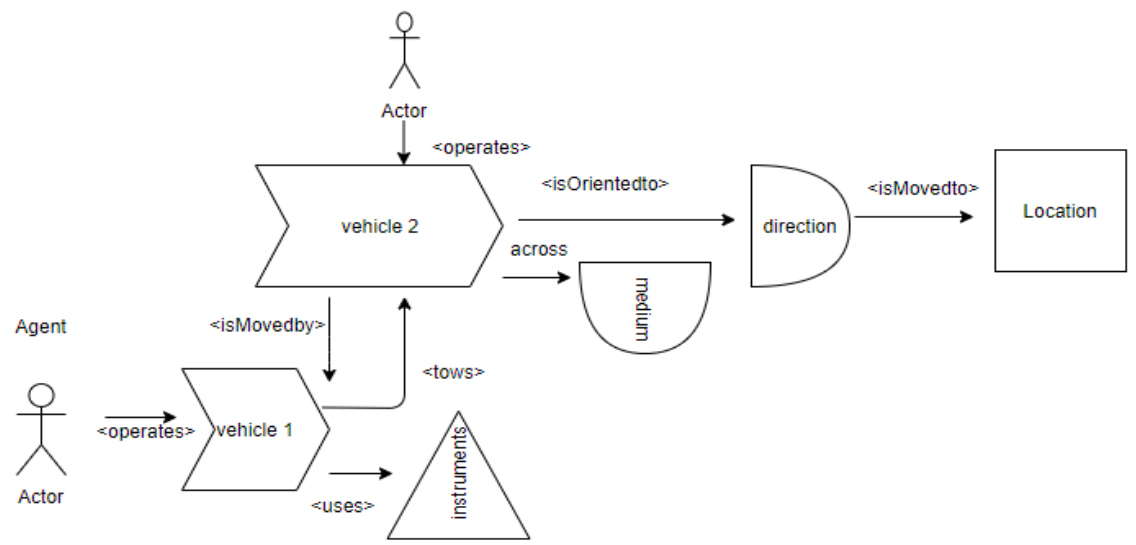

Figura 6: Diagrama de representación semántica del evento remolque maritimo

(Fuente: elaboración propia con el programa Online Visual Paradigm)

Como quedó establecido al principio, esta investigación puede considerarse como un primer paso hacia la preparación de un recurso término-ontológico que incluya entidades, propiedades, relaciones, equivalencias y definiciones. De esta forma, a través de su consulta, el traductor, intérprete, lingüista, profesor o el estudiante de inglés en el ámbito marítimo, podrá disponer, al mismo tiempo, de una visión específica y global del acontecimiento comunicativo del remolque marítimo. Podrá acceder al conocimiento especializado, es decir, a las acciones involucradas en el hecho del remolque (push/empujar, draw/arrastrar, pull/tirar), a las acciones colaterales que rodean al hecho del remolque (acciones a realizar conjuntamente por el buque remolcado), a los vehículos implicados, a los agentes implicados que gobiernan el evento del remolque marítimo en sus distintas etapas, a las secuencias de las acciones en el evento del remolque marítimo y a la instrumentación de la que se sirven para ejecutar las acciones del evento remolque. La creación de una término-ontología requiere, además de un entorno computacional en el que implementarla, de herramientas específicas para su construcción, de una técnica de modelado, de un lenguaje, de una metodología y de un estudio de la oración completa, no solo de segmentos oracionales. Su desarrollo podrá ser objeto de una investigación ulterior.

A pesar de que se han obtenido los objetivos marcados, se considera necesario avanzar en el estudio de las construcciones de verbo más partícula (CVP) y refinar las diferencias semánticas de casos en los que el grupo verbo+preposición está interceptado por una frase nominal. Sería conveniente completar las construcciones fraseológicas del evento del remolque maritimo que aglutina otros momentos como la maniobra de amarrar cabos en las distintas maquinarias de cubierta, así como valorar la disposición de los verbos en voz pasiva y si esta condiciona la interpretación de la acción. Asimismo, se propone para futuras investigaciones la incorporación de las unidades terminológicas sustantivas al estudio y su extensión a la 
construcción sintáctica de sujeto ya que es fundamental para completar el ciclo de conocimiento. Además, los resultados obtenidos con el corpus a partir de la búsqueda de towing muestran un potencial significativo en unidades terminológicas polilexemáticas sustantivas. Finalmente, es esencial la ampliación del corpus inglés Tug-Tow y la aportación de un corpus espanol comparable o paralelo para alinear las frases, proporcionar las equivalencias y contrastar la construcción sintáctica y semántica entre las lenguas.

Son varios los estudios que demuestran una relación entre la semántica del verbo y el contexto o paradigma sintáctico en el que se realiza. Sin embargo, hay verbos en los que esta relación no es directa y habría que continuar estudiando. Obtener datos en este sentido y en los distintos paradigmas de las lenguas especializadas contribuiria a su tratamiento en el terreno del procesamiento de lenguaje natural. Aplicado a nuestro objetivo ulterior, el desarrollo de un recurso término-ontológico en este campo, es primordial contar con la información semántica como paso previo, para acceder a perfilar la representación conceptual del dominio de la maniobra de remolque marítimo. En todo término ha de consignarse tanto la información conceptual como la información lingüística pues ambas son partes inherentes del mismo y todo recurso que complete esta información podrá, en principio, acercarse a responder a las necesidades del profesional de la lengua y del entorno marítimo.

\section{REFERENCIAS}

ANSTED, A. (1985): A Dictionary of Sea Terms. Glasgow: Brown, Son \& Fergurson, Limited.

ANTHONY, L. (2017): AntConc 3.5.0 [Computer Software]. Tokyo, Japan: Waseda University. https://www.laurenceanthony.net/software (Fecha de consulta: 09/01/2020).

BAISA, V., MOŽE, S. \& RENAU, I. (2016): "Linking Verb Pattern Dictionaries of English and Spanish", McCrae, J. et al. (eds.), Proceedings of the LREC 2016 Workshop. LDL 20165th Workshop on Linked Data in Linguistics: Managing, Building, and Using Linked Language Resources, Portorož, Slovenia: European Language Resources Association, pp. 41-45. http://www.lrecconf.org/proceeings/lrec2016/workshops/LREC2016Workshop-LDL2016_Proceedings.pdf (Fecha de consulta: 10/10/2020).
CLARK, E. V. \& CLARK, H. H. (1979): "When Nouns Surface as Verbs", Language, 55(4), pp. 767-811.

CRESPO RODRÍGUEZ, R. (1979): Vocabulario de construcción naval (español-inglés; inglés-español), Madrid: Fondo editorial de ingeniería naval. Asociación de ingenieros navales.

DROUIN, P. (2003): TermoStat 3.0 [Computer software]. http://termostat. ling.umontreal.ca/ (Fecha de consulta: $18 / 02 / 2020)$.

FILLMORE, C. J. (1982): "Frame Semantics", The Linguistic Society of Korea (ed.), Linguistics in the Morning Calm. Selected Papers from SICOL 1981, Seoul, Korea: Hanshin Publishing Company, pp. 11-137.

FRASER, B. (1976): The Verb-Particle Combination in English, New York, USA: Academic Press.

GOLDBERG, A. E. (1995): Constructions: A Construction Grammar Approach 
to Argument Structure, Chicago and London: The University of Chicago Press.

HANKS, P. (2008): "Lexical Patterns: From Hornby to Hunston and Beyond", Bernal, E. y DeCesaris, J. (eds.), Proceedings of the XIII EURALEX International Congress, Barcelona: Universitat Pompeu Fabra, pp. 89129. https://www.euralex.org/elx_proceedings/Euralex2008/004_Euralex_2008_Plenary_Patrick\%20Hanks_Lexical\%20Pat-

terns_from $\% 20$ Hornby $\% 20$ to $\% 20$ Huston\%20and\%20Beyond.pdf (Fecha de consulta: 16/04/2019).

HWANG, J. D. \& PALMER, M. (2015): "Identification of Caused Motion Construction", Palmer, M. Boleda, G. y Rosso, P. (eds.), Proceedings of the Fourth Joint Conference on Lexical and Computational Semantics, Denver, Colorado: The *SEM2015, pp. 51-60. https://www.aclweb.org//anthology/S15-1006.pdf (Fecha de consulta: 18/11/2019).

KAY, P. (2005): "Argument Structure Constructions and the Argument-Adjunct Distinction”, Fied, M. y Boas, H. C. (eds.), Grammatical Constructions. Amsterdam/Philadelphia: John Benjamins Company, pp. 71-98.

KIPPER SCHULER, K. (2005): VerbNet: A broad-coverage, comprehensive verb lexicon. $\mathrm{PhD}$. Thesis, Philadelphia: University of Pennsylvania. http://verbs.colorado.edu/ kipper/Papers/dissertation.pdf (Fecha de consulta: 15/01/2020).

KIPPER, K., DANG, H. T. \& PALMER, M. (2000): "Class-based Construction of a Verb Lexicon", Proceedings of the 17th National Conference on Artificial Intelligence (AAAI-OO), Austin, Texas: AAAI Press, pp. 691696. https://www.aaai.org/Papers/AAAI/2000/AAAIOO-

106.pdf. (Fecha de consulta: 26/11/2019).

KIPPER, K., KORHONEN, A., RYANT, N. \& PALMER, M. (2008): "A LargeScale Classification of English Verbs", Language Resources and
Evaluation, 42, pp. 21-40. doi 10.1007/s10579-007-9048-2. (Fecha de consulta: 13.12.2019).

KORHONEN, A. \& BRISCOE, T. (2004): "Extended Lexical-Semantic Classification of English Verbs", Proceedings of the HLT/NAACL Workshop on Computational Lexical Semantics, pp. 38-45. https://www.aclweb.org/anthology/W04-

2606.pdf (Fecha de consulta: 27/11/2019).

LEECH, G. (2004): Meaning and the English Verb, London: Pearson Education Ltd.

LEVIN, B. (1993): English Verb Classes and Alternations: A Preliminary Investigation, Chicago: University of Chicago Press.

LEVIN, B. \& RAPPAPORT HOVAV, M. (1995): Unaccusativity, Cambridge MA: MIT Press.

LEY 14/2014, DE 24 DE JULIO, DE NAVEGACIÓN MARÍTIMA: Boletín Oficial del Estado, núm. 180, de viernes 25 de julio de 2014, de 59193 a 59311 . https://www.boe .es/boe/dias/2014/07/25/pdfs/ BOE-A-2014-7877.pdf. (Fecha de consulta: 15/09/2019).

MILLER, G. A. (1995): “A Lexical Database for English", Communications of the ACM, 38(11), pp. 39-41.

PALMER, F. R. (1974): The English Verb, London: Longman.

PALMER, M., GILDEA, D. \& KINGSBURY, P. (2005): "The Proposition Bank: A Corpus Annotated with Semantic Roles", Computational Linguistics, 31(1), pp. 71-106.

PRINCETON UNIVERSITY. (2010). "About WordNet", WordNet, Princeton, New Jersey: Princeton University. Disponible en: https://wordnet.princeton.edu/ (Fecha de consulta: 20/03/2020).

REAL ACADEMIA ESPAÑOLA DE LA LENGUA (1726-1739): Diccionario de Autoridades, Tomo V (1737), Publicación electrónica: http:// web.frl.es/DA.html (Fecha de consulta: 22/01/2020).

REAL ACADEMIA ESPAÑOLA DE LA LENGUA. (2008): Nuevo Tesoro Lexicográfico de la Lengua Española. 
Publicación electrónica: http://ntlle.rae.es. (Fecha de consulta: 21/01/2020).

WRAY, A. (2000): "Formulaic Sequences in Second Language Teaching:
Principle and Practice", Applied Linguistics, 21(4), pp. 463-489.

YOUNG, A. (1863): Nautical Dictionary, London: Longman, Green, Longman, Roberts \& Green.

Anexo 1

\begin{tabular}{|c|c|c|}
\hline Verbo & Fraseologia verbal en inglés & $\begin{array}{l}\text { Índice de espe- } \\
\text { cificidad del } \\
\text { verbo base } \\
\text { constituyente }\end{array}$ \\
\hline To tow & $\begin{array}{c}\text { To tow from the stern } \\
\text { To tow from the bow } \\
\text { To tow a barge } \\
\text { To tow a dumb dredger } \\
\text { To tow other vessels } \\
\text { To tow ships off } \\
\text { To tow aft } \\
\text { To tow foreward } \\
\text { To tow the barge to } \\
\text { To tow alongside } \\
\text { To tow into position } \\
\text { To tow on the port quarter }\end{array}$ & 181.78 \\
\hline To moor & $\begin{array}{l}\text { To moor the vessel in } \\
\text { To moor facing east } \\
\text { To moor with both anchors }\end{array}$ & 73.26 \\
\hline To heave & $\begin{array}{c}\text { To heave the vessel into position } \\
\text { To heave the vessel out of his berth } \\
\text { To heave in the messenger line } \\
\text { To heave in the black rope } \\
\text { To heave in the anchors } \\
\text { To heave up the towline } \\
\text { To heave up the tow wire } \\
\text { To heave the towing hawser } \\
\text { To heave the vessel off with beach gear } \\
\text { To heave the vessel alongside }\end{array}$ & 30.5 \\
\hline To pull & $\begin{array}{c}\text { To pull astern } \\
\text { To pull down } \\
\text { To pul a ship } \\
\text { To pull the barge away } \\
\text { To pull the tug laterally } \\
\text { To pull the workboat along } \\
\text { To pull the stern away } \\
\text { To pull full } \\
\text { To pull against the fixed end of the brake } \\
\text { To be pulled over by its tow line }\end{array}$ & 20.56 \\
\hline To push & $\begin{array}{c}\text { To push the tug } \\
\text { To push the ship } \\
\text { To push a barge } \\
\text { To push stern against the ship } \\
\text { To push out the rope } \\
\text { To push away from } \\
\text { To push on the port quarter } \\
\text { To push the ship a bit } \\
\text { To push the messenger line back }\end{array}$ & 14.7 \\
\hline
\end{tabular}

Tabla 1: Extracto del inventario de construcciones fraseológicas verbales argumentativas en inglés del dominio de las maniobras de remolque maritimo basadas en el corpus Tug-Tow (Fuente: elaboración propia) 